\title{
A Continued Fraction Algorithm for the Computation of Higher Transcendental Functions in the Complex Plane
}

\author{
By I. Gargantini* and P. Henrici ${ }^{\dagger}$
}

\begin{abstract}
This report deals with the numerical evaluation of a class of functions of a complex variable that can be represented as Stieltjes transforms of nonnegative real functions. The considered class of functions contains, among others, the confluent hypergeometric functions of Whittaker and the Bessel functions. The method makes it possible, in principle, to compute the values of the function with an arbitrarily small error, using one and the same algorithm in whole complex plane cut along the negative real axis. Detailed numerical data are given for the application of the algorithm to the modified Bessel function $K_{0}(z)$.
\end{abstract}

1. Introduction. The present paper deals with the numerical evaluation of functions of a complex variable $z$ that can be represented in the form

$$
f(z)=\int_{0}^{\infty} \frac{d \psi(t)}{z+t}
$$

where the function $\psi$ is real, bounded, and nondecreasing with infinitely many points of increase, and where $z$ varies in the domain $G=\{z|z \neq 0,| \arg z \mid<\pi\}$ (i.e., in the complex plane cut along the negative real axis). Our method makes it possible, in principle, to calculate $f(z)$ with an arbitrarily small error, using one and the same algorithm for all $z \in G$. The algorithm automatically furnishes realistic a posteriori error estimates. Difficulties due to slow convergence and/or numerical instability must be expected if $z$ is close to the boundary of $G$, i.e., if $z$ is almost zero or real and negative.

It is not necessary for the application of the algorithm that the function $\psi$ be easily accessible. It may even be identical with the fraction which is to be computed. We require only that the integrals

$$
(-1)^{n} c_{n}=\int_{0}^{\infty} t^{n} d \psi(t), \quad n=0,1,2, \cdots
$$

all exist and be known with great precision (in practice this means that the coefficients $c_{n}$ can be expressed in analytic form). It is also required that $\left|c_{n}\right|$ does not grow too rapidly with $n$; a growth such that

$$
\sum_{n=0}^{\infty}\left|c_{n}\right|^{-1 / 2 n}=\infty
$$

is admissible. (Condition (3) is satisfied when $(-1)^{n} c_{n}=(n !)^{\alpha}$ if $\alpha \leqq 2$.)

Received March 14, 1966.

* IBM Zurich Research Laboratory, Switzerland.

$†$ Eidgenössische Technische Hochschule Zürich, Switzerland. 
2. An Example. A nontrivial example of a function to which our algorithm applies is given by the function defined for arbitrary real values of the parameters $\alpha$ and $\beta$ and for $\operatorname{Re} z>0$ by

$$
\Phi_{\alpha, \beta}(z)=\int_{0}^{\infty} e^{-s z}{ }_{2} F_{1}\left(\frac{1}{2}+\alpha, \frac{1}{2}+\beta ; 1 ;-s\right) d s .
$$

(Here ${ }_{2} F_{1}$ denotes the hypergeometric function of Gauss.) Alternate representations of this function, valid for $|\arg z|<\pi$, are

$$
\Phi_{\alpha, \beta}(z)=\frac{1}{z \Gamma\left(\frac{1}{2}+\beta\right)} \int_{0}^{\infty} e^{-t} t^{\beta-1 / 2}\left(1+\frac{t}{z}\right)^{-\alpha-1 / 2} d t \quad\left(\beta>-\frac{1}{2}\right)
$$

and a similar expression with $\alpha$ and $\beta$ interchanged.

The function $\Phi_{\alpha, \beta}$ is connected with Whittaker's confluent hypergeometric function $W_{k, \mu}$ through the relations

$$
\begin{aligned}
& \Phi_{\alpha, \beta}(z)=z^{(\alpha+\beta) / 2-1} e^{z / 2} W_{-(\alpha+\beta) / 2,(\beta-\alpha) / 2}(z), \\
& W_{k, \mu}(z)=z^{k+1} e^{-z / 2} \Phi_{\mu-k,-\mu-k}(z) .
\end{aligned}
$$

Like Whittaker's function, it is thus elementarily related to a number of other special functions of mathematical physics. For instance, as seen from (5), $\Phi_{1 / 2, \beta}$ essentially reduces to the incomplete $\Gamma$-function (and thus, if $\beta=0$, to the error integral). Furthermore, the modified Bessel function of the second kind can be expressed in terms of $\Phi_{\alpha, \beta}$ by means of the relation

$$
K_{\nu}(z)=(2 \pi z)^{1 / 2} e^{-z} \Phi_{\nu,-\nu}(2 z) .
$$

As is well known, the function $K_{\nu}$ occupies a central position in the theory of Bessel functions, since all other Bessel functions can be expressed through it. For instance, if $|\arg z|<\pi / 2$, and if we put $\omega_{\nu}=e^{i v \pi / 2}$, then

$$
\begin{aligned}
& J_{\nu}(z)=-\frac{1}{i \pi}\left[\omega_{\nu} K_{\nu}(i z)-\bar{\omega}_{\nu} K_{\nu}(-i z)\right], \\
& Y_{\nu}(z)=-\frac{1}{\pi}\left[\omega_{\nu} K_{\nu}(i z)+\bar{\omega}_{\nu} K_{\nu}(-i z)\right] .
\end{aligned}
$$

In particular, if $z=x>0$, then

$$
J_{\nu}(x)=-\frac{2}{\pi} \operatorname{Im} \omega_{\nu} K_{\nu}(i x), \quad Y_{\nu}(x)=-\frac{2}{\pi} \operatorname{Re} \omega_{\nu} K_{\nu}(i x) .
$$

Thus by evaluating $K_{\nu}(z)$ for the single complex number $i x$, we obtain at the same time the Bessel function of the first and of the second kind.

By (4), $\Phi_{\alpha, \beta}$ is merely the Laplace transform of ${ }_{2} F_{1}\left(\frac{1}{2}+\alpha, \frac{1}{2}+\beta ; 1 ;-s\right)$. By using known series expansions, it is easily shown that

$$
{ }_{2} F_{1}\left(\frac{1}{2}+\alpha, \frac{1}{2}+\beta ; 1 ;-s\right)=\frac{1}{\Gamma\left(\frac{1}{2}+\alpha\right) \Gamma\left(\frac{1}{2}+\beta\right)} \int_{0}^{\infty} e^{-s t} e^{-t} t^{\alpha+\beta} \Phi_{-\alpha_{0}-\beta}(t) d t .
$$

Substituting this expansion in (4) and interchanging the order of integrations, we obtain the crucial representation

$$
\Phi_{\alpha, \beta}(z)=\frac{1}{\Gamma\left(\frac{1}{2}+\alpha\right) \Gamma\left(\frac{1}{2}+\beta\right)} \int_{0}^{\infty} \frac{e^{-t} t^{\alpha+\beta} \Phi_{-\alpha,-\beta}(t)}{z+t} d t
$$


valid for $\alpha>-\frac{1}{2}, \beta>-\frac{1}{2}$. The representation (5) shows that $\Phi_{-\alpha,-\beta}(t)$ is positive for $t>0$ if at least one of the parameters $\alpha$ and $\beta$ is less than $\frac{1}{2}$. Trivially, this is also true for

$$
\Phi_{-\alpha,-1 / 2}(t)=\int_{0}^{\infty} e^{-t s} d s=\frac{1}{t} .
$$

Thus, the representation (9) is of the form (1), provided that $\alpha>-\frac{1}{2}, \beta>-\frac{1}{2}$, and that at least one of the parameters is contained in the interval $\left(-\frac{1}{2}, \frac{1}{2}\right]$. It follows from (4) that the coefficients $c_{n}$ are given by

$$
c_{n}=(-1)^{n} \frac{\left(\frac{1}{2}+\alpha\right)_{n}\left(\frac{1}{2}+\beta\right)_{n}}{n !} .
$$

By Stirling's formula, these coefficients are easily seen to satisfy (3).

3. The Algorithm. The basic idea of the algorithm has been given in [4]. By expanding $(z+t)^{-1}$ in powers of $z^{-1}$ and integrating term by term, it follows that the function $f$ defined by (1) possesses the asymptotic expansion

$$
f(z) \sim \sum_{n=0}^{\infty} \frac{c_{n}}{z^{n+1}}
$$

valid if $|z| \rightarrow \infty$ such that $|\operatorname{Im} z|$ is bounded away from zero. Alternatively, using the remainder term of the geometric series we have for $n=0,1,2, \cdots$

$$
f(z)=\frac{c_{0}}{z}+\frac{c_{1}}{z^{2}}+\cdots+\frac{c_{n-1}}{z^{n}}+\frac{c_{n}}{z^{n+1}} \vartheta_{n}(z),
$$

where

$$
\vartheta_{n}(z)=\frac{z}{c_{n}} \int_{0}^{\infty} \frac{(-t)^{n} d \psi(t)}{z+t} .
$$

The coefficient $\vartheta_{n}(z)$ is called the $n$th converging factor of the asymptotic expansion (11).

Our technique for evaluating $f(z)$ consists in expanding $\vartheta_{n}(z)$ in a continued fraction of the Stieltjes type and using the formula (12). Under the conditions stipulated in Section 1, the continued fraction converges for all $z \in G$. The choice of $n$ is, in principle, arbitrary; however, since the evaluation of $\vartheta(z)$ consumes more time than the summation of the terms $c_{k} z^{-k-1}$, it is recommended to choose $n$ such that $\left|c_{n} z^{-n-1}\right|$ is as small as possible, since then the permissible relative error in $\vartheta_{n}(z)$ is as large as possible.

The continued fraction representation of the converging factors $\vartheta_{n}(z)$ can be obtained, simultaneously for all values of $n$, from the quotient-difference (QD)scheme [8] associated with the series (11). This scheme is defined by the relations

$$
e_{0}^{(n)}=0, \quad q_{1}^{(n)}=\frac{c_{n+1}}{c_{n}} \quad n=0,1,2, \cdots
$$

and

$$
\begin{array}{lr}
e_{k}^{(n)}=\left[q_{k}^{(n+1)}-q_{k}^{(n)}\right]+e_{k-1}^{(n+1)}, & n=0,1,2, \cdots, \\
q_{k+1}^{(n)}=\frac{e_{k}^{(n+1)}}{e_{k}^{(n)}} q_{k}^{(n+1)}, & k=1,2, \cdots .
\end{array}
$$


It can be shown that under the conditions stated in Section 1, no zero divisors can occur, and the scheme is thus well defined. If the coefficients $q$ and $e$ are arranged in alternating columns, as shown in Table I, then each of the relations (14) connects 4 adjacent entries in an obvious fashion.

In terms of these coefficients, we have

$$
\vartheta_{n}(z)=\frac{z \mid}{\mid z}-\frac{q_{1}^{(n)} \mid}{\mid 1}-\frac{e_{1}^{(n)} \mid}{\mid z}-\frac{q_{2}^{(n)} \mid}{\mid 1}-\frac{e_{2}^{(n)} \mid}{\mid z}-\cdots, \quad n=0,1,2, \cdots
$$

which may be written in the equivalent form

$$
\vartheta_{n}(z)=\frac{\zeta \mid}{\mid \zeta}+\frac{a_{2}^{(n)} \mid}{\mid \zeta}+\frac{a_{3}^{(n)} \mid}{\mid \zeta}+\frac{a_{4}^{(n)} \mid}{\mid \zeta}+\cdots,
$$

where $\zeta=z^{1 / 2}, \operatorname{Re} \zeta>0$, and

$$
a_{2 k}^{(n)}=-q_{k}^{(n)}, \quad a_{2 k+1}^{(n)}=-e_{k}^{(n)} .
$$

The fraction (15b) is evaluated numerically as the limit of its approximants

$$
w_{k}=\rho_{k} / \sigma_{k}
$$

as $k \rightarrow \infty$, where $\rho_{k}$ and $\sigma_{k}$ are defined by the recurrence relations

$$
\begin{array}{lll}
\rho_{0}=0, & \rho_{1}=\zeta, & \rho_{k}=a_{k}^{(n)} \rho_{k-2}+\zeta \rho_{k-1},
\end{array} \quad k=2,3, \cdots .
$$

4. An Estimate for the Truncation Error. Even if truncated, the continued fraction (15a) or the equivalent fraction (15b) conveys accurate information about the location of the complex number $\vartheta_{n}(z)$. The following theorem is proved in [5]:

THEOREM. Let $w_{-1}=\infty, w_{0}=0, w_{1}, w_{2}, \cdots$, denote the approximants of the continued fraction $(15 \mathrm{~b})$, and let $\gamma_{m}(m=0,1,2, \cdots)$ denote the circular arc originating at $w_{m-1}$, passing through $w_{m+1}$, and terminating at $w_{m}$. Then, for each $m=$ $1,2, \cdots$, the value of (15b) is contained in the compact point set $\Omega_{m}$ bounded by $\gamma_{m}$ and by that portion of $\gamma_{m-1}$ which lies between $w_{m-1}$ and $w_{m}$.

It is also known that the sets $\Omega_{m}$ are convex, that the two circular arcs bounding. them always intersect at an angle $|\arg z|$, that $\Omega_{m+1} \subset \Omega_{m}(m=1,2, \cdots)$, and that the diameter of $\Omega_{m}$ tends to zero for $m \rightarrow \infty$. For $z$ real and positive, the arcs

\section{TABLE I}

$$
\mathbf{0}
$$

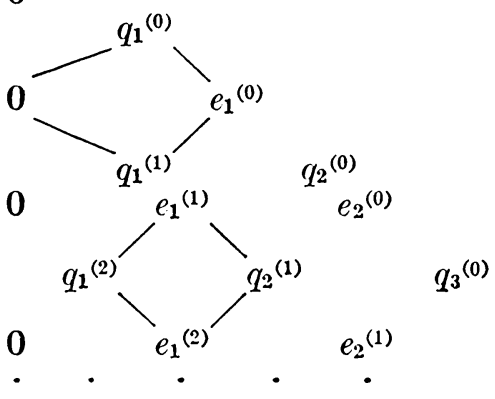




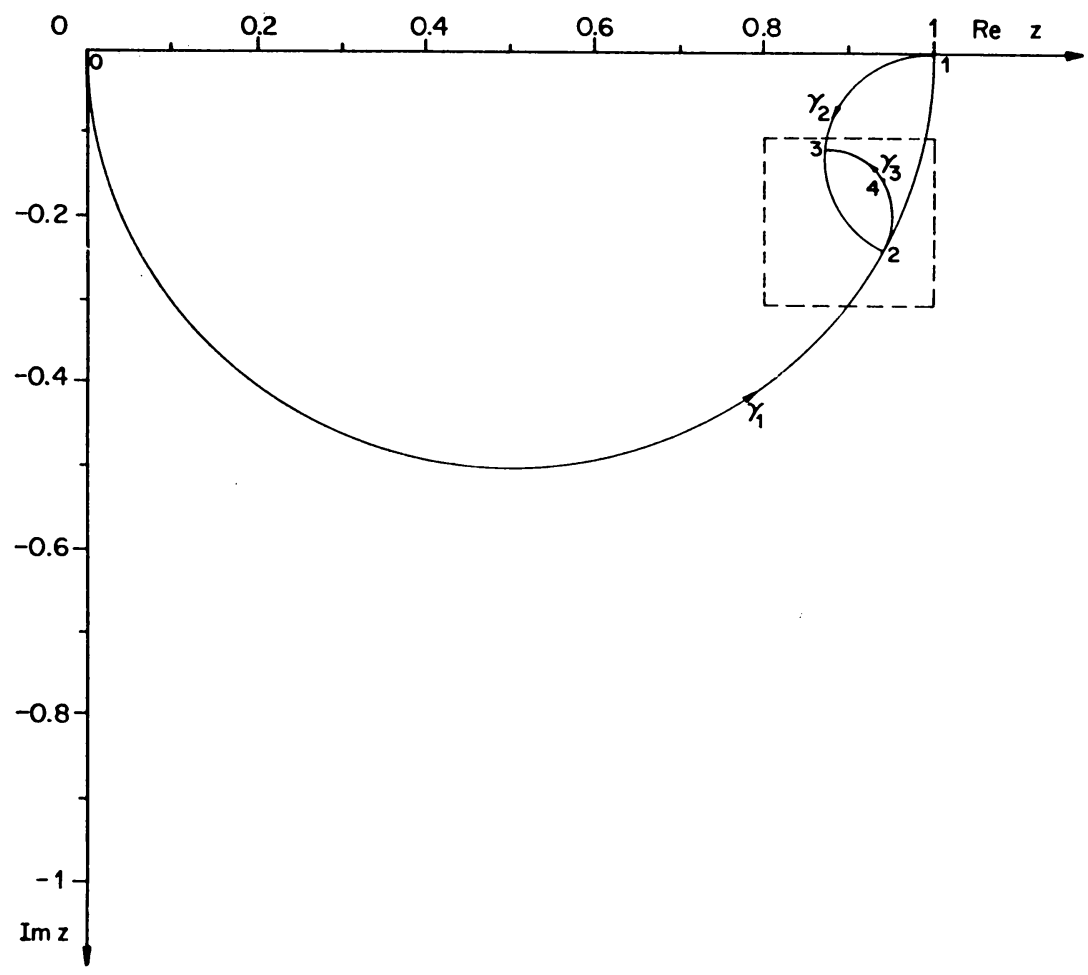

Fig. 1a

$\gamma_{m}$ and the sets $\Omega_{m}$ degenerate into straight line segments. It is well known in this case that $\vartheta_{n}(z)$ is contained in the interval spanned by any two consecutive approximants.

In Figs. $1 \mathrm{a}$ and $1 \mathrm{~b}$ we show the $\operatorname{arcs} \gamma_{m}$ and the sets $\Omega_{m}$ for the case $z=-i / 2$ of the continued fraction representation for the converging factor $\vartheta_{0}(z)$ of the series

$$
\sum_{n=0}^{\infty} \frac{(-1)^{n}\left[\left(\frac{1}{2}\right)_{n}\right]^{2}}{2^{n} n ! z^{n+1}}
$$

asymptotic to:

$$
f(z)=e^{z} \sqrt{\frac{2}{\pi z}} K_{0}(z) .
$$

5. Construction of the QD-Scheme. In a few cases of interest it is possible to give explicit formulas for the elements of the QD-scheme associated with the series (11). For example, for the function $\Phi_{1 / 2, \beta}$ related to the incomplete $\Gamma$-function we have

hence

$$
c_{n}=(-1)^{n}\left(\frac{1}{2}+\beta\right)_{n},
$$

$$
q_{1}^{(n)}=-\left(\beta+n+\frac{1}{2}\right),
$$

and thus, as is easily verified,

$$
\begin{aligned}
& e_{k}^{(n)}=-k, \\
& q_{k}^{(n)}=-\left(\beta+n+k-\frac{1}{2}\right)
\end{aligned}
$$




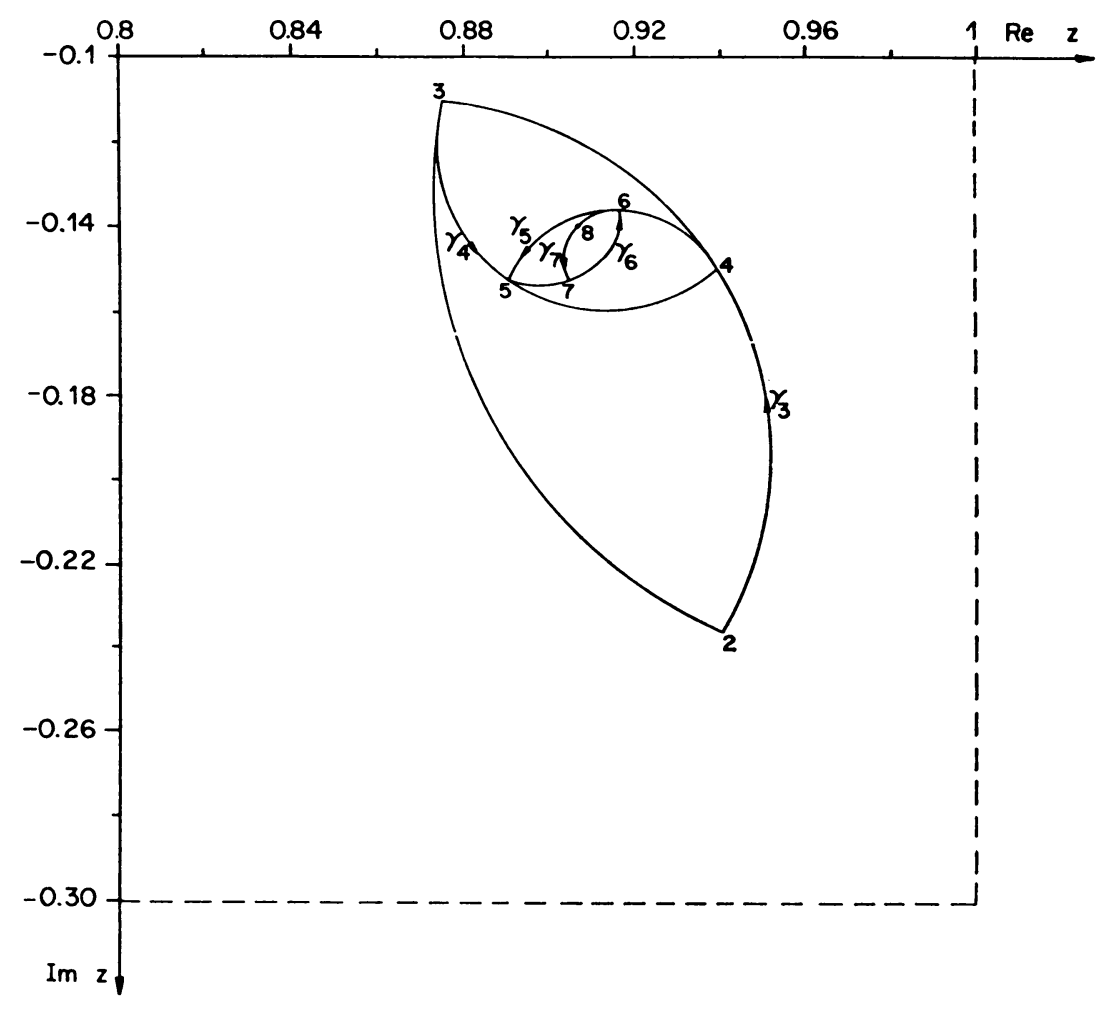

Fig. 1b

$(n=0,1,2, \cdots ; k=1,2, \cdots)$. The resulting continued fractions for $\vartheta_{n}(z)$ are essentially known (at least for $n=0$, see [6], p. 148 or [7], p. 103) and have been used for numerical purposes with good results [1].

In many other cases of interest, no such explicit formulas are known, and it becomes necessary to construct the QD-scheme numerically. This numerical construction is not without hazards in view of the well-known numerical instability of the QD-algorithm. The reason for the instability can easily be seen from the above example: For $k$ fixed, $q_{k}^{(n)}$ grows linearly with $n$. The first formula (14) thus requires forming the difference of two nearly equal large numbers, with obviously ensuing loss of accuracy. It thus becomes necessary to look for other, more stable, methods for generating the scheme. We shall discuss several possible approaches, only one of which proved to be practically successful.

5.1. Rational Arithmetic. If the coefficients $c_{n}$ are rational, all entries of the QD-scheme are rational, and it is theoretically possible to construct the scheme in rational arithmetic by treating every number as an ordered pair of integers (see [3]). This technique was found impractical, even in a simple case such as (17), because the number of digits in both numerator and denominator increases very rapidly with increasing $k$, although every fraction was reduced to lowest terms by means of the Euclidian algorithm. Using a maximum word length of 50 decimal digits on the IBM 1620 it was possible to compute the QD-scheme for $K_{0}(z)$ only up to $k=4$. In another experiment, the QD-scheme associated with the function 
$J(z)=\log \Gamma(z)-\left(z-\frac{1}{2}\right) \log z+z-\frac{1}{2} \log 2 \pi$ (where the $c_{n}$ are related to the Bernoulli numbers), the calculations could only be carried out up to $k=3$. To the extent to which they were computed, the number thus obtained could be used as a standard against which values obtained by other methods could be compared.

5.2. Progressive Form of Algorithm. In some applications of the QD theory (for instance, when the algorithm is used for the factorization of polynomials, see [8]), the algorithm can be stabilized if the scheme is generated row by row instead of column by column. The necessary first two rows can be obtained from the series $\sum d_{n} z^{-n-1}=z^{-2}\left[\sum c_{n} z^{-n-1}\right]^{-1}$, whose coefficients $d_{n}$ can be found from the $c_{n}$ via simple recurrence relations. In order to find out whether a similarly stabilizing effect takes place also in the present context, we applied this method to the series $\sum(-1)^{n} n ! z^{-n-1}$, where the entries of the scheme (for $n \geqq 0$ ) are given by $(19 b)$ if $\beta=\frac{1}{2}$. However, this method turned out to be just about as unstable as the method of generating the scheme column by column. What seems to happen here is that the ratios $d_{n+1} / d_{n}$ behave asymptotically exactly like the ratios $c_{n+1} / c_{n}$. It would be interesting to have theoretical confirmation of this experimental observation.

5.3. Incremental Form of Algorithm. The basic idea here is as follows: If, for given values $c_{n}$, the first quotients $q_{1}^{(n)}$ differ only slightly from (19a), the resulting entries in the scheme will differ only little from (19b). A little more generally, we assume that

$$
q_{1}{ }^{(n)}=A n+B+\gamma_{1}^{(n)}
$$

where $A$ and $B$ are constants and where the $\gamma_{1}{ }^{(n)}$ are "small". Writing

$$
\begin{aligned}
& {q_{k}}^{(n)}=A(n+k-1)+B+\gamma_{k}{ }^{(n)}, \\
& e_{k}^{(n)}=k A+\epsilon_{k}^{(n)},
\end{aligned}
$$

we find that the "corrections" $\gamma_{k}^{(n)}$ and $\epsilon_{k}^{(n)}$ satisfy the recurrence relations $\epsilon_{0}^{(n)}=0$,

$$
\begin{aligned}
& \epsilon_{k}^{(n)}=\left(\gamma_{k}^{(n+1)}-\gamma_{k}^{(n)}\right)+\epsilon_{k-1}^{(n+1)}, \\
& \gamma_{k+1}^{(n)}=\gamma_{k}^{(n+1)}+\left(\epsilon_{k}^{(n+1)}-\epsilon_{k}^{(n)}\right) \frac{(n+k) A+B+\gamma_{k}^{(n+1)}}{k A+\epsilon_{k}^{(n)}}
\end{aligned}
$$

$(n=0,1,2, \cdots ; k=1,2, \cdots)$, which are somewhat more complicated than (14) but which no longer involve differences of large quantities.

In our standard example $f(z)=e^{z} \sqrt{(2 / \pi z)} K_{0}(z)$, where

we find that

$$
c_{n}=(-1)^{n} \frac{\left[\left(\frac{1}{2}\right)_{n}\right]^{2}}{2^{n} n !} \text {, }
$$

$$
q_{1}^{(n)}=-\frac{(2 n+1)^{2}}{8(n+1)}=-\frac{1}{2} n-\frac{1}{8(n+1)},
$$

and (20) thus holds with $A=-\frac{1}{2}, B=0, \gamma_{1}{ }^{(n)}=-1 / 8(n+1)$. More generally, if the $c_{n}$ are the coefficients of the asymptotic series for $\Phi_{\alpha, \beta}(\lambda z)$,

$$
c_{n}=(-1)^{n} \frac{\left(\frac{1}{2}+\alpha\right)_{n}\left(\frac{1}{2}+\beta\right)_{n}}{n ! \lambda^{n+1}},
$$




\begin{tabular}{|c|c|}
\hline ब्త్ & 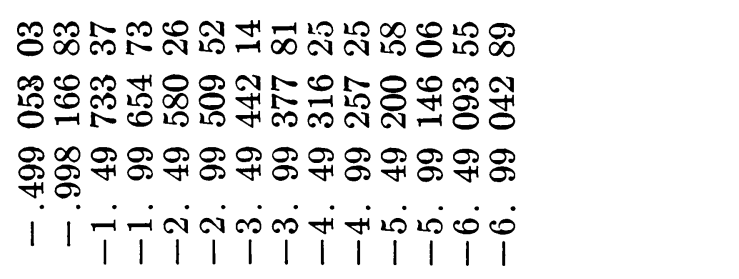 \\
\hline @ & 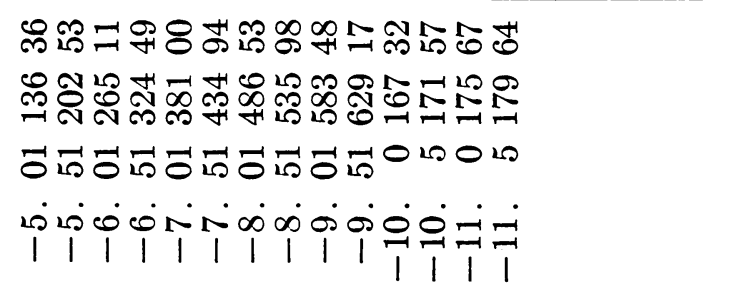 \\
\hline$\widehat{\varrho}$ & 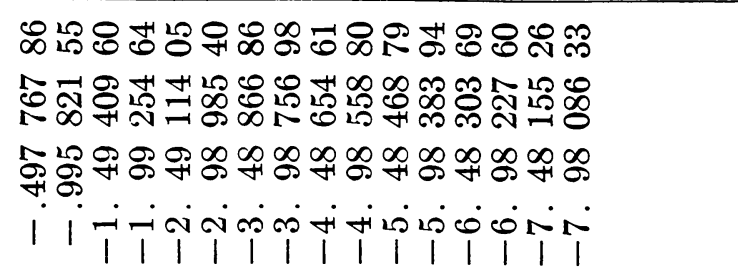 \\
\hline$\stackrel{0}{\sigma}$ & 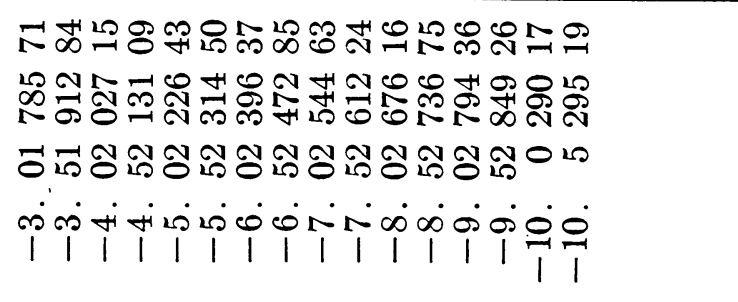 \\
\hline$\widehat{\mathrm{a}}$ & 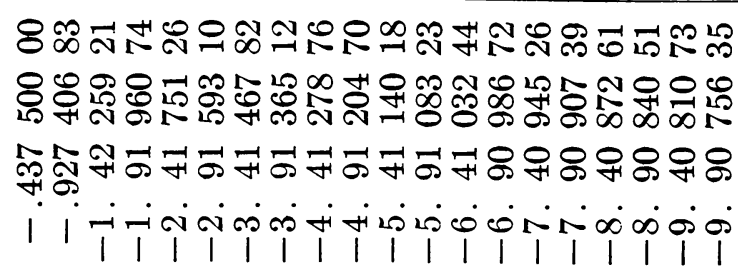 \\
\hline$\hat{a}^{\circ}$ & 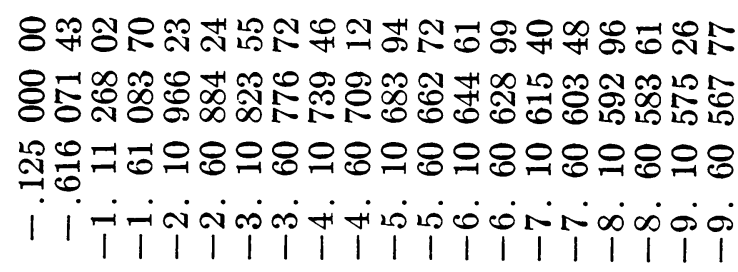 \\
\hline$\leqslant$ & ーN⿻ \\
\hline
\end{tabular}


then

$$
\begin{aligned}
{q_{1}}^{(n)} & =-\frac{\left(\alpha+n+\frac{1}{2}\right)\left(\beta+n+\frac{1}{2}\right)}{\lambda(n+1)} \\
& =-\frac{1}{\lambda} n-\frac{\alpha+\beta}{\lambda}-\frac{\left(\alpha-\frac{1}{2}\right)\left(\beta-\frac{1}{2}\right)}{\lambda(n+1)},
\end{aligned}
$$

which again is of the form (20). Quite generally it can be shown that if

$$
\gamma_{1}{ }^{(n)} \sim \sum_{k=1}^{\infty} \frac{C_{k}}{n^{k}}
$$

as $n \rightarrow \infty$, then all $\gamma_{k}{ }^{(n)}$ and $\epsilon_{k}{ }^{(n)}$ possess asymptotic expansions as $n \rightarrow \infty$, with the leading terms

$$
\gamma_{k}^{(n)} \sim C_{1} / n, \quad \epsilon_{k}{ }^{(n)} \sim-k C_{1} / n^{2} .
$$

Our initial assumption about the smallness of the corrections is thus justified.

Although much more stable than the original form of the algorithm, the above "incremental" version is not yet perfectly stable due to the fact that the fraction in the second formula (22) is comparable to $n / k$ for large values of $n$. If $u$ denotes

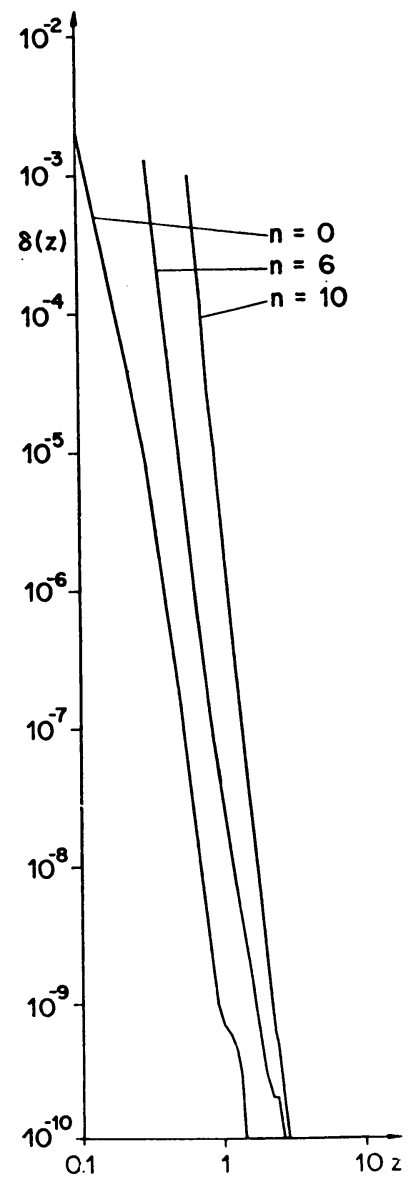

FIG. 2 
the basic unit of the machine number representing the mantissa of a floating number, a crude analysis using the formulas (24) shows that the error in $\gamma_{k}{ }^{(n)}$ may be as large as

$$
\left|\frac{2^{2 k-4}}{n^{2}}\left(\begin{array}{c}
n+k-1 \\
k-1
\end{array}\right) \cdot u C_{1}\right| .
$$

However, due to statistical effects the actual error can be expected to be much smaller.

We have used the above method to compute the QD-scheme associated with $K_{0}(z)$ [whose first $q$-column is given by (23)] for $n=0, k=20$; for $n=1,2 \cdots$, $40, k=10-[n / 2]$. The corrections $\gamma_{k}^{(n)}$ and $\epsilon_{k}^{(n)}$ were computed from (22) and the entries of the scheme subsequently from (21). The computations were performed in both double and triple precision. All 24 decimal digits of the triple precision values agreed with the values computed in rational arithmetic wherever the latter were available (i.e., up to $k=4$ ). The double precision values agreed with the triple precision values up to $k=10(n=0,1, \cdots, 19)$. Double precision

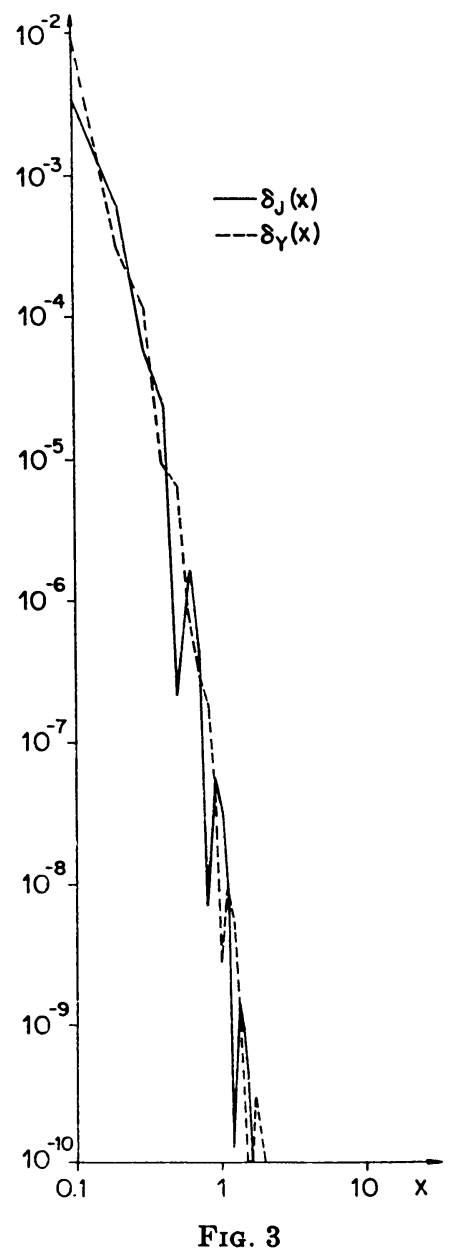


values computed directly from (14) agreed with the triple precision values only up to $k=7(n=0,1, \cdots, 25)$.

Table II presents the coefficients $q_{k}, e_{k}$ of the continued fractions $\vartheta_{0}(z), \vartheta_{6}(z)$, $\vartheta_{10}(z)$ associated with $K_{0}(z)$.

6. Numerical Results for $K_{0}(z)$. Having discussed the construction of the QD-scheme pertaining to the function $K_{0}(z)$, we shall now present some data concerning the evaluation of the function itself. As indicated above, the method of evaluation consists in using the representation

$$
K_{0}(z)=e^{-z} \sqrt{\frac{\pi z}{2}}\left\{\frac{c_{0}}{z}+\frac{c_{1}}{z^{2}}+\cdots+\frac{c_{n-1}}{z^{n}}+\frac{c_{n}}{z^{n+1}} \vartheta_{n}(z)\right\},
$$

where

$$
c_{k}=(-1)^{k} \frac{\left[\left(\frac{1}{2}\right)_{k}\right]^{2}}{2^{k} k !}=(-1)^{k} \frac{1^{2} 3^{2} \cdots(2 k-1)^{2}}{2^{3 k} k !},
$$

and where $\vartheta_{n}(z)$ is the continued fraction (15a) calculated via the QD-scheme from the power series $\sum c_{n} z^{-n-1}$ as described in Section 5.3.

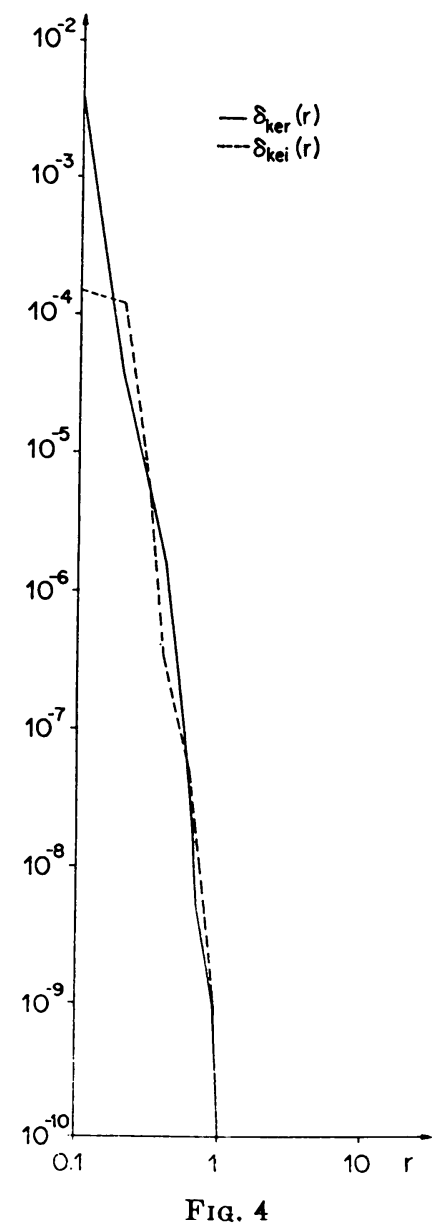


In order to test the algorithm in a realistic manner, the triple precision values of $q_{k}{ }^{(n)}$ and $e_{k}{ }^{(n)}$ were truncated to single precision. The remaining operations, however, were carried out in double precision, mainly to avoid rounding error in forming the partial sums of the asymptotic expansion.

Fig. 2 shows the accuracy of the evaluation of $K_{0}(z)$ for $z>0$. The absolute value of the difference between the values of $e^{z} K_{0}(z)$, computed for $z=0.1(0.1) 2(1) 10$ and the values given in the tables [2] are plotted in a logarithmic scale. The converging factors $\vartheta_{0}, \vartheta_{6}, \vartheta_{10}$ (with $k_{\max }=20,16,14$, respectively) were used. For small values of $z(z<2$, say), it is evidently best to transform the whole asymptotic into a continued fraction, i.e., to work with $n=0$.

Purely imaginary values of $z$ and values with argument $\pi / 4$ were also tried. These values can be checked by means of the relations

$$
\begin{aligned}
K_{0}(-i x) & =\frac{i \pi}{2}\left[J_{0}(x)+i Y_{0}(x)\right], \\
K_{0}\left(r e^{i(\pi / 4)}\right) & =\operatorname{ker}(r)+i k e i(r)
\end{aligned}
$$

(see [9], p. 78 and p. 81).

For clarity, only the results for $n=0$ are presented in Figs. 3 and 4. Although theoretically the convergence of the continued fraction is worse than for real $z$, the results are virtually the same in all three cases: For $|z| \geqq 1$, the continued fraction permits an evaluation of $K_{0}(z)$ with an error of at most $10^{-9}$.

IBM Zurich Research Laboratory

8803 Rüschlikon-ZH, Switzerland

1. S. D. Conte \& B. D. Fried, The Plasma Dispersion Function, Academic Press, New York, 1961.

2. Handbook of Mathematical Functions, National Bureau of Standards Mathematics Series, No. 55, Government Printing Office, Washington, D. C., 1964.

3. P. HenRICI, "A subroutine for computations with rational numbers", J. Assoc. Comput. Mach., v. 3, 1956, pp. 6-9. MR 17, 673.

4. P. Hen RICI, Some A pplications of the Quotient-Difference Algorithm, Proc. Sympos. Appl.

Math. Vol. 15, Amer. Math. Soc., Providence, R. I., 1963, pp. 159-183. MR 28 \#2632.

5. P. Henrici \& P. Pfluger, "Truncation error estimate for Stieltjes fractions," (To appear.)

6. A. N. Khovanskil, The Application of Continued Fractions and Their Generalizations to Problems in Approximation Theory, Noordhoff, Groningen, 1963. MR 27 \#6058.

7. O. Perron, Die Lehre von den Kettenbrüchen. Vol. 2: Analytischfunktionentheoretische Kettenbrüche, 3rd ed., Teubner, Stuttgart, 1957. MR 19, 25.

8. H. RUTISHAUSER. "Der Quotienten-Differenzen-Algorithmus," Mitt. Inst. Angew. Math. Zürich, no. 7, MR 19, 686.

9. G. N. Watson, $A$ Treatise on the Theory of Bessel Functions, 2nd ed. Cambridge Univ. Press, Cambridge, 1962. 